\title{
A United Method for Sensitivity Analysis of the Locational Marginal Price Based on the Optimal Power Flow
}

\author{
Liu Yang ${ }^{1}$ and Chunlin Deng ${ }^{2}$ \\ ${ }^{1}$ School of Mathematics and Computational Science, Xiangtan University, Xiangtan, Hunan 411105, China \\ ${ }^{2}$ Department of Public Management, Xiangtan University, Xiangtan 411105, China \\ Correspondence should be addressed to Liu Yang; yangl410@163.com
}

Received 15 June 2014; Accepted 4 August 2014; Published 18 August 2014

Academic Editor: Chunlin Chen

Copyright (c) 2014 L. Yang and C. Deng. This is an open access article distributed under the Creative Commons Attribution License, which permits unrestricted use, distribution, and reproduction in any medium, provided the original work is properly cited.

\begin{abstract}
Locational marginal prices (LMPs) are influenced by various factors in the electricity market; knowing the sensitivity information of LMPs is very important for both the purchase and the consumer. This paper presents a united method to compute the sensitivities of LMPs based on the optimal power flow (OPF). The Karush-Kuhn-Tucher (KKT) system to solve LMPs can be transferred into an equation system by using an NCP function, and then by using the properties of the derivative of the semismooth NCP function, this paper provides a simultaneous obtention of the sensitivities of LMPs with respect to power demands, the cost of production, voltage boundary, and so forth. Numerical examples illustrate the concepts presented and the proposed methodology by a 6-bus electric energy system. Some relevant conclusions are drawn in the end.
\end{abstract}

\section{Introduction}

In electricity market, LMPs can reflect the actual cost of electricity supply from both space and time, and it has governed the electricity business. It has now been implemented or scheduled for implementation in U.S. energy regions in the midwest (MISO), New England (ISO-NE), New York (NYISO), the Mid-Atlantic states (PJM), California (CAISO), the southwest (SPP), and Texas (ERCOT) [1-4]. China is building east power market with this pricing mechanism. Therefore, more and more researchers began to focus on the pricing mechanisms [1-6].

As is well known, LMPs are dual variables arising in OPF problems, conditional on a given network topology. Specifically, they are the shadow prices corresponding to the nodal balance constraints that impose Kirchhoffs circuit law at each bus. In the short run, when fuel prices and other supply conditions are relatively stable, load is one of the most significant factors driving the determination of LMPs. So within an optimal power flow (OPF) framework, LMPs are the sensitivities (dual variables) associated with the active power balance equations $[4,7,8]$. When LMPs govern the electricity business, a fundamental question is how LMPs change as parameters change. These dual variables might require adjustments to be interpreted as LMPs within some market clearing frameworks, and the parameters which influence LMPs in the power market include the load demand, production costs, and voltage boundary.

The changes in LMPs as parameters vary provide insight on the functioning and behavior of the electric energy system. This sensitivity information might help producers and consumers to establish their respective bidding strategies and the regulator to assess the degree of competitiveness of the electricity market. However, we only consider the local sensitivities which provide information for small changes, not for large changes in this paper.

In the literature, sensitivity calculations are generally related to the power flow problem $[9,10]$ or the OPF problem involving sensitivities of the objective function and the primal variables with respect to parameters [8]. In [7], the sensitivities of certain primal variables (power injections) with respect to certain dual variables (LMPs) are calculated. But the objective function is considered linear and only equality constraints are taken into account. References $[6,8]$ provide a perturbation approach to sensitivity analysis similar to the one presented in $[11,12]$ and apply it to the OPF problem. 
Within a vertically integrated electric utility framework, paper [13] uses the results of [8] to carry out a valuation of the transmission impact in a resource bidding process. Reference [14] builds a system model to quantitatively analyze the effects of various factors such as load uncertainty, thermal limit, capacity reserve, market power, and their interactions on the mean and standard deviation of the LMPs. Reference [15] gives a global analysis of system variable sensitivities when LMPs are derived from DCOPF solutions for dayahead energy market. All the existing work only considered the LMPs sensitivities with respect to several variables in different cases. However, in this paper, we will calculate the sensitivities of all dual variables with respect to all parameters of the problem in a general nonlinear programming case including equality and inequality constraints. We use the results reported in [16] and give a new expression to calculate the sensitivities based on the semismooth KKT system.

The construction of this paper is as follows. In Section 2, we give the marginal price mathematical model and define the sensitivity of the LMPs based on OPF. In Section 3, we transfer the KKT system of the OPF problem into a system of semismooth equations and present the local sensitivity formulas for both the cases of regular nondegenerate and the cases of regular degenerate solutions. In Section 4, numerical examples are given by an electricity system with 6 nodes. In Section 5, we get some conclusions of this paper.

\section{Mathematical Model of the LMPs Based on OPF}

In a competitive electricity market, the settlement between the independent system operator (ISO) and the participants is based on locational marginal prices (LMPs). LMP at a given node of a power system is the sensitivity of operational cost to the change in load at that node, and it is calculated using an optimal power flow (OPF) program. When LMPs are used for settlement of transactions, consumers are charged more than the average cost of production of electricity due to the nonlinear nature of the power flow and the constraints imposed by the OPF. So mathematical model to calculate the LMPs is as follows [4].

2.1. The Objective Function (Generation Cost Function). In electricity market, in addition to the active production costs, the production cost of the reactive power should also be considered in this model, so the active and reactive power cost function with a quadratic function curve is

$$
\min _{p_{G}, q_{G}, v, \delta} z=f\left(p_{G}, q_{G}, v, \delta, p_{D}, q_{D}, c\right),
$$

where $p_{G}$ is the active power generation, $q_{G}$ is the reactive power generation, $v$ is the node voltage amplitude, $\delta$ is the phase angle, $p_{D}$ is the active load demand, $q_{D}$ is the reactive load demand, and $c$ indicates all other parameters associated with the network and generator.
2.2. Equality Constraints (The Power Balance Equation). Imposed by the OPF, we should consider the following equality constraints:

$$
\begin{aligned}
& p_{G_{i}}-p_{D_{i}}-h_{p_{i}}\left(p_{G}, q_{G}, v, \delta, p_{D}, q_{D}, c\right)=0, \quad i=1,2, \ldots, n_{B}, \\
& q_{G_{i}}-q_{D_{i}}-h_{Q_{i}}\left(p_{G}, q_{G}, v, \delta, p_{D}, q_{D}, c\right)=0, \quad i=1,2, \ldots, n_{B},
\end{aligned}
$$

where (2) are active and reactive power flow equations.

\subsection{The Inequality Constraints}

(a) Line flow constraints:

$$
\begin{array}{r}
-\left(s_{i j}^{\max }\right)^{2}+\left(g_{s_{i j}}\left(p_{G}, q_{G}, v, \delta, p_{D}, q_{D}, c\right)\right)^{2} \leq 0, \\
i=1,2, \ldots, n_{B}, \forall j \in \Omega_{i},
\end{array}
$$

where $s_{i j}^{\max }$ means the transmission capacity on the line $i j, g_{s_{i j}}$ is a composite power value on the line $i j, n_{B}$ represents the total number of nodes, and $\Omega_{i}$ is the related node connecting node $i$.

(b) Capacity constraints:

$$
\begin{array}{ll}
p_{G}^{\min }-p_{G} \leq 0, & p_{G}-p_{G}^{\max } \leq 0, \\
q_{G}^{\min }-q_{G} \leq 0, & p_{G}-p_{G}^{\max } \leq 0 .
\end{array}
$$

(c) Voltage constraints:

$$
v^{\min }-v \leq 0, \quad v-v^{\max } \leq 0 .
$$

(d) Voltage angles constraint:

$$
-\delta-\pi \leq 0, \quad \delta-\pi \leq 0 .
$$

For the sake of simplicity, it is assumed that all the needs are not resilient. In order to derive the sensitivities of problem (1)-(6), we transfer it into the following simply nonlinear programming problems (NLPP):

$$
\begin{array}{cc}
\min _{x} & z=f(x, a), \\
\text { s.t. } & h(x, a)=0, \\
& g(x, a) \leq 0,
\end{array}
$$

where $h(x, a)=\left(h_{1}(x, a), \ldots, h_{l}(x, a)\right)^{T}, g(x, a)=$ $\left(g_{1}(x, a), \ldots, g_{m}(x, a)\right)^{T}, \quad x \in R^{n}$ includes all the optimal variables $v, \delta, p_{G}, q_{G}$, and $a \in R^{p}$ includes all the parameters $p_{D}, q_{D}, c$.

\section{Derivation of the Sensitivity Formulas}

In this section, we derive local sensitivity formulas of the LMPs. Note that the LMPs are the Lagrange multiplier vectors for the active power flow equations. So we can derive the KKT system of problem (7)-(9) firstly. 
3.1. Semismooth Equations Equivalent to the KKT Systems of Problem. Let $x^{*}$ be a local optimal solution, and we assume the linear independent constraint qualification is satisfied. From the first-order optimal condition, there exists a pair of vectors $\lambda^{*} \in R^{l}$ and $\mu^{*} \in R^{m}$ satisfying the following KKT systems $[11,12]$ :

$$
\begin{gathered}
\nabla_{x} f\left(x^{*}, a\right)+\sum_{k=1}^{l} \lambda_{k}^{*} \nabla_{x} h_{k}\left(x^{*}, a\right) \\
+\sum_{j=1}^{m} \mu_{j}^{*} \nabla_{x} g_{j}\left(x^{*}, a\right)=0_{n}, \\
h_{k}\left(x^{*}, a\right)=0, \quad k=1,2, \ldots, l, \\
g_{j}\left(x^{*}, a\right) \leq 0, \quad j=1,2 \ldots, m, \\
\mu_{j}^{*} g_{j}\left(x^{*}, a\right)=0, \quad j=1,2, \ldots, m, \\
\mu^{*} \geq 0_{m},
\end{gathered}
$$

where $\lambda^{*}$ and $\mu^{*}$ are the KKT multipliers, also known as the dual variables.

To obtain the sensitivity analysis, we perturb or modify $x^{*}, \sigma, \lambda^{*}, \mu^{*}, z^{*}$ in such a way that the KKT systems (10) still hold. Thus, we will usually differentiate the objective function (7) and the KKT systems (10) directly. But in this kind of method, one must deal with a lot of compositions of equalities and inequalities in the case of regular degenerate solution (in this case, there is at least one dual variable $\mu_{j}^{*}=0$ ), and the calculation and analysis become much complex. So in this paper, we will transfer the KKT systems (10) into a system of semismooth equations by using an NCP function; then, we can get the united formulas to calculate the local sensitivity for both the case of regular nondegenerate solution (in this case, the dual variable $\mu_{j}^{*} \neq 0$ ) and the case of regular degenerate solution by using the semismooth properties.

Let $\varphi: R^{2} \rightarrow R$ be Fischer-Burmeister function (see [17]); the definition is

$$
\varphi(a, b)=\sqrt{a^{2}+b^{2}}-(a+b) .
$$

It is easy to see that $\varphi(a, b)=0$ if and only if

$$
a \geq 0, \quad b \geq 0, \quad a^{T} b=0 .
$$

A function with this property is called an NCP function. For the sake of convenience, we denote the Fischer-Burmeister function as FB function. From [17], we know that the FB function is differentiable everywhere except at the point $(0,0)$ and it is semismooth at $(0,0)$. The definition and properties of semismooth function can be found in [18].
By using the FB function, we transfer the KKT system (10) into a system of semismooth equations:

$$
\begin{gathered}
\nabla_{x} f\left(x^{*}, \bar{\sigma}\right)-\sum_{k=1}^{l} \lambda_{k}^{*} \nabla_{x} h_{k}\left(x^{*}, \bar{\sigma}\right) \\
-\sum_{j=1}^{m} \mu_{j}^{*} \nabla_{x} g_{j}\left(x^{*}, \bar{\sigma}\right)=0_{n}, \\
h_{k}\left(x^{*}, \bar{\sigma}\right)=0, \quad k=1,2, \ldots, l, \\
\phi\left(x^{*}, \mu^{*}, \bar{\sigma}\right)=0_{m},
\end{gathered}
$$

where

$$
\begin{aligned}
& \phi\left(x^{*}, \mu^{*}, \bar{\sigma}\right) \\
& \quad=\left(\phi_{1}\left(x^{*}, \mu^{*}, \bar{\sigma}\right), \phi_{2}\left(x^{*}, \mu^{*}, \bar{\sigma}\right), \ldots, \phi_{m}\left(x^{*}, \mu^{*}, \bar{\sigma}\right)\right)^{T}, \\
& \phi_{j}\left(x^{*}, \mu^{*}, \bar{\sigma}\right) \\
& \quad=\varphi\left(\mu_{j}^{*}, g_{j}\left(x^{*}, \bar{\sigma}\right)\right) \\
& \quad=\mu_{j}^{*}+g_{j}\left(x^{*}, \bar{\sigma}\right)-\sqrt{\left(\mu_{j}^{*}\right)^{2}+\left(g_{j}\left(x^{*}, \bar{\sigma}\right)\right)^{2}}, \\
& \quad j=1,2, \ldots, m .
\end{aligned}
$$

3.2. Local Sensitivity Formulas for the Case of Regular Nondegenerate Solution. In this case, the dual variable $\mu_{j}^{*} \neq 0$. From the properties of the FB function, we know that $\phi_{j}\left(x^{*}, \mu^{*}, \sigma\right)$ is continuously differentiable. So we differentiate the object function (7) and the KKT system (10) as follows:

$$
\begin{gathered}
\left(\nabla_{x} f\left(x^{*}, \bar{\sigma}\right)\right)^{T} d x+\left(\nabla_{\sigma} f\left(x^{*}, \bar{\sigma}\right)\right)^{T} d \sigma-d z=0, \\
\left(\nabla_{x x} f\left(x^{*}, \bar{\sigma}\right)-\sum_{k=1}^{l} \lambda_{k}^{*} \nabla_{x x} h_{k}\left(x^{*}, \bar{\sigma}\right)\right. \\
\left.-\sum_{j=1}^{m} \mu_{j}^{*} \nabla_{x x} g_{j}\left(x^{*}, \bar{\sigma}\right)\right) d x \\
+\left(\nabla_{x \sigma} f\left(x^{*}, \bar{\sigma}\right)-\sum_{k=1}^{l} \lambda_{k}^{*} \nabla_{x \sigma} h_{k}\left(x^{*}, \bar{\sigma}\right)\right. \\
\left.-\sum_{j=1}^{m} \mu_{j}^{*} \nabla_{x \sigma} g_{j}\left(x^{*}, \bar{\sigma}\right)\right) d \sigma \\
-\nabla_{x} h\left(x^{*}, \bar{\sigma}\right) d \lambda-\nabla_{x} g\left(x^{*}, \bar{\sigma}\right) d \mu=0_{n} \\
\left(\nabla_{x} h\left(x^{*}, \bar{\sigma}\right)\right)^{T} d x+\left(\nabla_{\sigma} h\left(x^{*}, \bar{\sigma}\right)\right)^{T} d \sigma=0_{l}, \\
\left(\nabla_{x} \phi\left(x^{*}, \mu^{*}, \bar{\sigma}\right)\right)^{T} d x+\left(\nabla_{\mu} \phi\left(x^{*}, \mu^{*}, \bar{\sigma}\right)\right)^{T} d \mu \\
\left.+\left(x^{*}, \mu^{*}, \bar{\sigma}\right)\right)^{T} d \sigma=0_{m},
\end{gathered}
$$


where $I=\left\{j=1, \ldots, m \mid g_{j}\left(x^{*}, a\right)=0\right\}, N=\{1, \ldots, m\} \backslash I$,

$$
\begin{gathered}
\nabla_{x} \phi_{j}\left(x^{*}, \mu^{*}, \bar{\sigma}\right)= \begin{cases}\nabla_{x} g_{j}\left(x^{*}, \bar{\sigma}\right), & j \in I, \\
0, & j \in N .\end{cases} \\
\nabla_{\mu} \phi_{j}\left(x^{*}, \mu^{*}, \bar{\sigma}\right)= \begin{cases}0, & j \in I, \\
1, & j \in N .\end{cases} \\
\nabla_{\sigma} \phi_{j}\left(x^{*}, \mu^{*}, \bar{\sigma}\right)= \begin{cases}\nabla_{\sigma} g_{j}\left(x^{*}, \bar{\sigma}\right), & j \in I, \\
0, & j \in N .\end{cases}
\end{gathered}
$$

Denote

$$
\begin{gathered}
F_{x}=\nabla_{x} f\left(x^{*}, \bar{\sigma}\right), \quad F_{\sigma}=\nabla_{\sigma} f\left(x^{*}, \bar{\sigma}\right), \\
F_{x x}=\nabla_{x x} f\left(x^{*}, \bar{\sigma}\right)-\sum_{k=1}^{l} \lambda_{k}^{*} \nabla_{x x} h_{k}\left(x^{*}, \bar{\sigma}\right) \\
-\sum_{j=1}^{m} \mu_{j}^{*} \nabla_{x x} g_{j}\left(x^{*}, \bar{\sigma}\right), \\
F_{x \sigma}=\nabla_{x \sigma} f\left(x^{*}, \bar{\sigma}\right)-\sum_{k=1}^{l} \lambda_{k}^{*} \nabla_{x \sigma} h_{k}\left(x^{*}, \bar{\sigma}\right) \\
-\sum_{j=1}^{m} \mu_{j}^{*} \nabla_{x \sigma} g_{j}\left(x^{*}, \bar{\sigma}\right), \\
H_{x}=\left(\nabla_{x} h\left(x^{*}, \bar{\sigma}\right)\right)^{T}, \quad H_{\sigma}=\left(\nabla_{\sigma} h\left(x^{*}, \bar{\sigma}\right)\right)^{T}, \\
G_{x}=\left(\nabla_{x} g\left(x^{*}, \bar{\sigma}\right)\right)^{T}, \quad \phi_{x}=\left(\nabla_{x} \phi\left(x^{*}, \mu^{*}, \bar{\sigma}\right)\right)^{T}, \\
\phi_{\sigma}=\left(\nabla_{\sigma} \phi\left(x^{*}, \mu^{*}, \bar{\sigma}\right)\right)^{T}, \quad \phi_{\mu}=\left(\nabla_{\mu} \phi\left(x^{*}, \mu^{*}, \bar{\sigma}\right)\right)^{T} .
\end{gathered}
$$

In matrix form, the system (15) can be written as

$$
\left[\begin{array}{ccccc}
F_{x} & F_{\sigma} & \mathbf{0} & \mathbf{0} & -1 \\
F_{x x} & F_{x \sigma} & -H_{x}^{T} & -G_{x}^{T} & \mathbf{0} \\
H_{x} & H_{\sigma} & \mathbf{0} & \mathbf{0} & \mathbf{0} \\
\phi_{x} & \phi_{\sigma} & \mathbf{0} & \phi_{\mu} & \mathbf{0}
\end{array}\right]\left[\begin{array}{l}
d x \\
d \sigma \\
d \lambda \\
d \mu \\
d z
\end{array}\right]=\mathbf{0} .
$$

Obviously, (18) is a system of linear equation, so we can get all the sensitivities from (18) easily. Furthermore, (18) can be written as

$$
U[d x, d \lambda, d \mu, d z]^{T}=S d \sigma,
$$

where $U, S$ are

$$
U=\left[\begin{array}{cccc}
F_{x} & \mathbf{0} & \mathbf{0} & -1 \\
F_{x x} & -H_{x}^{T} & -G_{x}^{T} & \mathbf{0} \\
H_{x} & \mathbf{0} & \mathbf{0} & \mathbf{0} \\
\phi_{x} & \mathbf{0} & \phi_{\mu} & \mathbf{0}
\end{array}\right], \quad S=-\left[\begin{array}{c}
F_{\sigma} \\
F_{x \sigma} \\
H_{\sigma} \\
\phi_{\sigma}
\end{array}\right] .
$$

It is obvious that $U$ is a square matrix and we can easily get the sensitivities of LMPs with respect to all parameters according to formula (19). The condition to ensure the application of (19) can be seen in [16].
3.3. Local Sensitivity Formulas for the Case of Regular Degenerate Solution. In this case, there exists the case of $\mu_{j}^{*}=$ $g_{j}\left(x^{*}, \bar{\sigma}\right)=0, j \in I_{0}=\left\{j \in I \mid \mu_{j}^{*}=0\right\}$. So $\phi_{j}\left(x^{*}, \mu^{*}, \sigma\right)$ is not differentiable but semismooth at this time. From the semismooth properties of

$$
\begin{aligned}
& \varphi\left(\mu_{j}^{*}, g_{j}\left(x^{*}, \bar{\sigma}\right)\right) \\
& \quad=\mu_{j}^{*}+g_{j}\left(x^{*}, \bar{\sigma}\right)-\sqrt{\left(\mu_{j}^{*}\right)^{2}+\left(g_{j}\left(x^{*}, \bar{\sigma}\right)\right)^{2}},
\end{aligned}
$$

we know that if the changes of $\mu_{j}^{*}$ or $g_{j}\left(x^{*}, \bar{\sigma}\right)$ (denoted by $d \mu_{j}^{*}$ or $\left.d g_{j}\right)$ are not equal to zero, we have

$$
\begin{aligned}
\phi_{j} & \left(x^{*}+d x, \mu^{*}+d \mu, \bar{\sigma}+d \sigma\right)-\phi_{j}\left(x^{*}, \mu^{*}, \bar{\sigma}\right) \\
& =\varphi\left(\mu^{*}+d \mu, g_{j}\left(x^{*}+d x, \bar{\sigma}+d \sigma\right)\right)-\varphi\left(\mu^{*}, g_{j}\left(x^{*}, \bar{\sigma}\right)\right) \\
& =V_{j x} d x+V_{j a} d \sigma+V_{j \mu} d \mu+o(\|(d x, d \sigma, d \mu)\|),
\end{aligned}
$$

where $I_{+}=I \backslash I_{0}=\left\{j \in I \mid \mu_{j}^{*}>0\right\}$, $V_{j x}=\nabla_{x} g_{j}\left(x^{*}, \bar{\sigma}\right)\left(1-\left(d g_{j} / \sqrt{\left(d g_{j}\right)^{2}+\left(d \mu_{j}\right)^{2}}\right)\right), V_{j \sigma}=$ $\nabla_{\sigma} g_{j}\left(x^{*}, \bar{\sigma}\right)\left(1-\left(d g_{j} / \sqrt{\left(d g_{j}\right)^{2}+\left(d \mu_{j}\right)^{2}}\right)\right)$, and $V_{j \mu}=1-\left(d \mu_{j} /\right.$ $\left.\sqrt{\left(d g_{j}\right)^{2}+\left(d \mu_{j}\right)^{2}}\right)$.

So in the case of regular degenerate solution, if $d \mu_{j}^{*}$ or $d g_{j}$ is not equal to zero and we aim to perturb $x^{*}, \sigma, \lambda^{*}, \mu^{*}, z^{*}$ in such a way that the KKT systems still hold, we have

$$
\begin{aligned}
& \left(\nabla_{x} f\left(x^{*}, \bar{\sigma}\right)\right)^{T} d x+\left(\nabla_{\sigma} f\left(x^{*}, \bar{\sigma}\right)\right)^{T} d \sigma-d z=0, \\
& \left(\nabla_{x x} f\left(x^{*}, \bar{\sigma}\right)-\sum_{k=1}^{l} \lambda_{k}^{*} \nabla_{x x} h_{k}\left(x^{*}, \bar{\sigma}\right)\right. \\
& \left.-\sum_{j=1}^{m} \mu_{j}^{*} \nabla_{x x} g_{j}\left(x^{*}, \bar{\sigma}\right)\right) d x \\
& +\left(\nabla_{x \sigma} f\left(x^{*}, \bar{\sigma}\right)-\sum_{k=1}^{l} \lambda_{k}^{*} \nabla_{x \sigma} h_{k}\left(x^{*}, \bar{\sigma}\right)\right. \\
& \left.\quad-\sum_{j=1}^{m} \mu_{j}^{*} \nabla_{x \sigma} g_{j}\left(x^{*}, \bar{\sigma}\right)\right) d \sigma \\
& -\nabla_{x} h\left(x^{*}, \bar{\sigma}\right) d \lambda-\nabla_{x} g\left(x^{*}, \bar{\sigma}\right) d \mu=0_{n} \\
& \left(\nabla_{x} h\left(x^{*}, \bar{\sigma}\right)\right)^{T} d x+\left(\nabla_{\sigma} h\left(x^{*}, \bar{\sigma}\right)\right)^{T} d \sigma=0_{l}, \\
& \left(\nabla_{x} \phi\left(x^{*}, \mu^{*}, \bar{\sigma}\right)\right)^{T} d x+\left(\nabla_{\mu} \phi\left(x^{*}, \mu^{*}, \bar{\sigma}\right)\right)^{T} d \mu \\
& +\left(\nabla_{\sigma} \phi\left(x^{*}, \mu^{*}, \bar{\sigma}\right)\right)^{T} d \sigma=0,
\end{aligned}
$$


where

$$
\begin{gathered}
\nabla_{x} \phi_{j}\left(x^{*}, \mu^{*}, \bar{\sigma}\right)= \begin{cases}\nabla_{x} g_{j}\left(x^{*}, \bar{\sigma}\right), & j \in I_{+}, \\
V_{j x}, & j \in I_{0}, \\
0, & j \in N .\end{cases} \\
\nabla_{\mu} \phi_{j}\left(x^{*}, \mu^{*}, \bar{\sigma}\right)= \begin{cases}0, & j \in I_{+}, \\
V_{j \mu}, & j \in I_{0}, \\
1, & j \in N .\end{cases} \\
\nabla_{\sigma} \phi_{j}\left(x^{*}, \mu^{*}, \bar{\sigma}\right)= \begin{cases}\nabla_{\sigma} g_{j}\left(x^{*}, \bar{\sigma}\right), & j \in I_{+}, \\
V_{j \sigma}, & j \in I_{0}, \\
0, & j \in N .\end{cases}
\end{gathered}
$$

Though (23) is not a system of linear equations, we can get the corresponding values of $V_{j x}, V_{j \sigma}, V_{j \mu}$ according to the adjustment of the active inequality with $\left(\mu_{j}^{*}, g_{j}\left(x^{*}, \sigma\right)\right)=$ $(0,0)$. We consider two cases as follows.

(i) If we want this inequality to remain active, we take $d g_{j}=0, d \mu_{j}>0$, so we get $V_{j x}=\nabla_{x} g_{j}\left(x^{*}, \bar{\sigma}\right), V_{j \mu}=0$, and $V_{j \sigma}=\nabla_{\sigma} g_{j}\left(x^{*}, \bar{\sigma}\right)$.

(ii) If we want this inequality to become inactive, we can take $d \mu_{j}=0, d g_{j}>0$, and get $V_{j x}=0, V_{j \mu}=1$, and $V_{j \sigma}=0$.

So we can also get linear equations to calculate all the sensitivities for the case of regular degenerate solution according to the adjustment of the active inequality with $\left(\mu_{j}^{*}, g_{j}\left(x^{*}, \sigma\right)\right)=(0,0)$ as follows:

$$
\left[\begin{array}{cccccc}
F_{x} & F_{\sigma} & \mathbf{0} & \mathbf{0} & \mathbf{0} & -1 \\
F_{x x} & F_{x \sigma} & -H_{x}^{T} & -G_{I^{\prime} x}^{T} & \mathbf{0} & \mathbf{0} \\
H_{x} & H_{\sigma} & \mathbf{0} & \mathbf{0} & \mathbf{0} & \mathbf{0} \\
G_{I^{\prime} x} & G_{I^{\prime} \sigma} & \mathbf{0} & \mathbf{0} & \mathbf{0} & \mathbf{0} \\
\mathbf{0} & \mathbf{0} & \mathbf{0} & \mathbf{0} & I_{N}^{\prime} & \mathbf{0}
\end{array}\right]\left[\begin{array}{c}
d x \\
d \sigma \\
d \lambda \\
d \mu \\
d z
\end{array}\right]=\mathbf{0},
$$

where $G_{I^{\prime} x}$ and $G_{I^{\prime} \sigma}$ represent the partial derivatives with respect to $x$ and $a$ of all the active inequalities which include the corresponding inequalities with $\left(\mu_{j}, g_{j}\left(x^{*}, \bar{\sigma}\right)\right)=(0,0)$ but are required to remain active. $I_{N}^{\prime}$ is the unit matrix whose cardinality is equal to the number of all inactive inequalities which include the corresponding inequalities with $\left(\mu_{j}, g_{j}\left(x^{*}, \bar{\sigma}\right)\right)=(0,0)$ but are required to become inactive. The condition to ensure the application of (25) can be seen in [16].

Expressions (19) and (25) allow deriving sensitivities of the LMPs with respect to active and reactive power demands. The simplicity of expressions (19) and (25) should be noted. The computational complexity of building matrices and evaluating expressions (19) and (25) is moderate even for large scale electric energy systems.

\section{Numerical Example}

The 6-bus electric energy system shown in Figure 1 is taken from [6]. The OPF model of this problem is

$$
\begin{aligned}
& \min _{p_{G_{i}}, q_{G_{i}}, i=1, \ldots, 3 ; v_{j}, \delta_{j}, j=1, \ldots, 6} \sum_{i=1}^{3}\left(a_{i} p_{G_{i}}+b_{i} p_{G_{i}}^{2}\right) \\
& p_{G_{i}}-p_{D_{i}} \\
& =v_{i} \sum_{j=1}^{6} v_{j}\left(G_{i j} \cos \left(\delta_{i}-\delta_{j}\right)+B_{i j} \sin \left(\delta_{i}-\delta_{j}\right)\right): \lambda_{i}, \\
& i=1, \ldots, 6 \text {; } \\
& q_{G_{i}}-q_{D_{i}} \\
& =v_{i} \sum_{j=1}^{6} v_{j}\left(G_{i j} \sin \left(\delta_{i}-\delta_{j}\right)-B_{i j} \cos \left(\delta_{i}-\delta_{j}\right)\right), \\
& i=1, \ldots, 6 \text {; } \\
& \left(v_{i} v_{j}\left(G_{i j} \cos \left(\delta_{i}-\delta_{j}\right)+B_{i j} \sin \left(\delta_{i}-\delta_{j}\right)\right)-G_{i j} v_{i}^{2}\right)^{2} \\
& +\left(v_{i} v_{j}\left(G_{i j} \sin \left(\delta_{i}-\delta_{j}\right)-B_{i j} \cos \left(\delta_{i}-\delta_{j}\right)\right)\right. \\
& \left.-v_{i}^{2}\left(B_{i j}-\frac{B_{i j}^{S}}{2}\right)\right)^{2} \\
& \leq\left(s_{i j}^{\max }\right)^{2}, \quad i=1, \ldots, 6, j \in \Omega_{i} \text {; } \\
& p_{G_{i}}^{\min } \leq p_{G_{i}} \leq p_{G_{i}}^{\max }, \quad i=1, \ldots, 3 ; \\
& q_{G_{i}}^{\min } \leq q_{G_{i}} \leq q_{G_{i}}^{\max }, \quad i=1, \ldots, 3 ; \\
& v^{\min } \leq v_{i} \leq v^{\max }, \quad i=1, \ldots, 6 ; \\
& -\pi \leq \delta_{i} \leq \pi, \quad i=2, \ldots, 6 \text {; } \\
& \delta_{1}=0 \text {, }
\end{aligned}
$$

where $a_{i}, b_{i}, G_{i j}, B_{i j}, \delta_{i}-\delta_{j}, B_{i j}^{S}, s_{i j}^{\max }$ are the linear cost coefficient of generator $i$, the quadratic cost coefficient of generator $i$, the element of the real part of the admittance matrix, the element of the imaginary part of the admittance matrix, the charging susceptance of line $i j$, and the transmission capacity of line $i j$, respectively. The optimal solution of the OPF above is illustrated in Table 1. Other data are given in Tables 2 and 3 , respectively, where the unit of $p_{G_{i}}^{*}$ is $\mathrm{MW}$, the unit of $q_{G_{i}}^{*}$ is MVAr, the unit of $v_{i}^{*}$ is p.u., the unit of $\delta_{i}^{*}$ is rad, and the unit of $\lambda_{p_{i}}^{*}$ is S/MWh. And $v^{\min }=0.9$ and $v^{\max }=1.1$. 


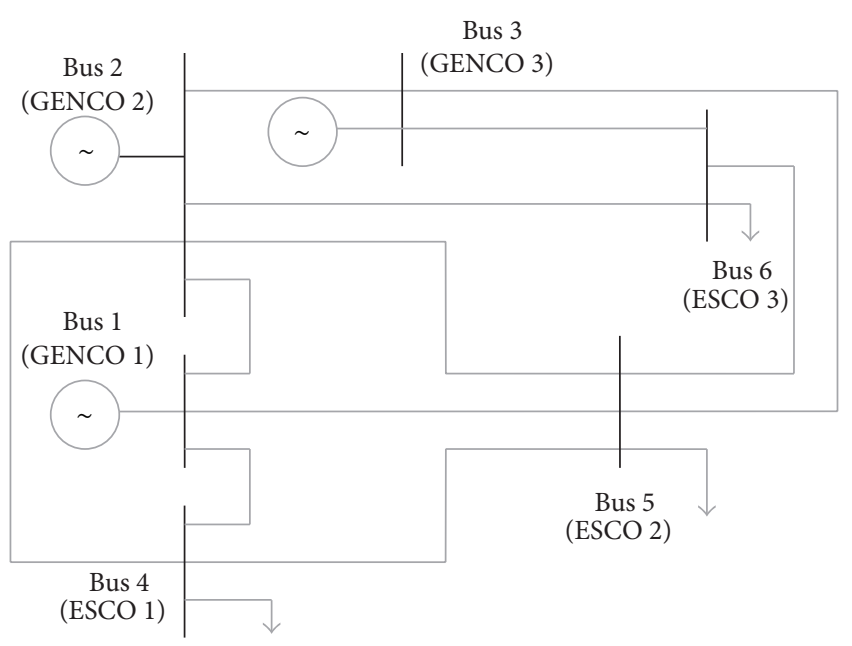

FIGURE 1: 6-bus electric energy system.

TABLE 1: OPF solution.

\begin{tabular}{lccccc}
\hline Bus & $p_{G_{i}}^{*}$ & $q_{G_{i}}^{*}$ & $v_{i}^{*}$ & $\delta_{i}^{*}$ & $\lambda_{P_{i}}^{*}$ \\
\hline 1 & 132.5 & 37.3 & 1.100 & 0.000 & 8.977 \\
2 & 160.6 & 92.9 & 1.100 & -0.047 & 9.161 \\
3 & 60.0 & 82.8 & 1.098 & -0.091 & 9.43 \\
4 & - & - & 1.018 & -0.091 & 9.733 \\
5 & - & - & 1.006 & -0.121 & 9.866 \\
6 & - & - & 1.034 & -0.128 & 9.711 \\
\hline
\end{tabular}

Because the optimal solution is known and it is a regular nondegenerate solution, we can get the sensitivities of the LMPs of the active load demand by using formula (19):

$$
\left[\frac{\partial \lambda_{i}}{\partial p_{D_{i}}}\right]=\left[\begin{array}{llllll}
2.162 & 0.098 & 0.492 & 3.852 & 1.610 & 0.639 \\
0.098 & 0.100 & 0.103 & 0.106 & 0.108 & 0.106 \\
0.492 & 0.103 & 0.843 & 1.023 & 0.412 & 0.644 \\
3.852 & 0.106 & 1.032 & 9.014 & 3.271 & 1.327 \\
1.610 & 0.108 & 0.412 & 3.271 & 2.124 & 0.701 \\
0.639 & 0.106 & 0.644 & 1.327 & 0.701 & 0.847
\end{array}\right]
$$

where $i$ is row and $j$ is column.

As can be seen from the above data, sensitivity coefficients of the LMPs with respect to all load demands are positive; they indicate that the LMPs and the load demands are changing in the same direction. Because generator 2 is the marginal generator, values in column/row 2 are small and close to 0.1 . The derivative of the LMP in bus 2 with respect to the demand in that bus equals the derivative with respect to demand of the marginal cost of the generator in that bus, because this generator is the swing generator. The highest price sensitivity occurs in bus 4 . This indicates that this bus might suffer a comparatively significant price volatility. Observe that the high price volatility of bus 4 is not a trivial property of the system, thus demonstrating the added value of the proposed analysis.
Sensitivities of LMPs with respect to reactive power demands are provided in the matrix below. Units are (\$/MWh)/(puMVAr):

$$
\left[\frac{\partial \lambda_{i}}{\partial q_{D_{i}}}\right]=\left[\begin{array}{cccccc}
0 & 0 & 0 & 2.135 & 0.666 & 0.170 \\
0 & 0 & 0 & 0.005 & 0.005 & 0.003 \\
0 & 0 & 0 & 0.551 & -0.091 & -0.040 \\
0 & 0 & 0 & 5.215 & 1.530 & 0.379 \\
0 & 0 & 0 & 1.910 & 1.033 & 0.293 \\
0 & 0 & 0 & 0.750 & 0.188 & 0.076
\end{array}\right] .
$$

We can observe that these sensitivities are much smaller than the sensitivities with respect to active power demands. Note that the first three columns of the matrix of LMPs derivatives with respect to reactive power demands are zero because reactive power has no cost (in this particular example) and is in between its bounds for each of the generators at the first three buses.

Sensitivities with respect to the voltage single upper bound and sensitivities with respect to generator (linear and quadratic) cost parameters $a_{j}$ and $b_{j}$ are provided by the vectors below:

$$
\begin{gathered}
{\left[\frac{\partial \lambda_{i}}{\partial v^{\max }}\right]=\left[\begin{array}{l}
-1.758 \\
-0.034 \\
-1.041 \\
-6.501 \\
-3.761 \\
-1.941
\end{array}\right], \quad\left[\frac{\partial \lambda_{i}}{\partial a_{j}}\right]=\left[\begin{array}{lll}
0 & 0.980 & 0 \\
0 & 1.000 & 0 \\
0 & 1.029 & 0 \\
0 & 1.063 & 0 \\
0 & 1.077 & 0 \\
0 & 1.060 & 0
\end{array}\right],} \\
{\left[\frac{\partial \lambda_{i}}{\partial b_{j}}\right]=\left[\begin{array}{lll}
0 & 314.9 & 0 \\
0 & 321.4 & 0 \\
0 & 330.8 & 0 \\
0 & 341.4 & 0 \\
0 & 346.1 & 0 \\
0 & 340.6 & 0
\end{array}\right]}
\end{gathered}
$$

As it can be seen, sensitivities with respect to the voltage single lower bound are zero, as no lower bound limit is reached for voltages. Sensitivities of LMPs with respect to cost coefficients are zero for generators working at their respective maximum or minimum power output. Note also that the derivative of the LMP in bus 2 with respect to the linear cost term of the generator in that bus is 1 because that generator is the swing generator. Finally, we can see that the sensitivities with respect to line design parameters (resistance, reactance, susceptance, and capacity) and generator design parameters (capacity, minimum power output, and cost parameters) are also readily available.

\section{Conclusion}

Within an OPF framework, this paper provides a new calculation method for the sensitivities of the LMPs. We analyze changes in LMPs with respect to operational parameters such as demands, generator cost parameters, and voltage bounds. However, the changes with respect to line design parameters such as resistance, reactance, susceptance, and capacity and generator design parameters including capacity and minimum power output can be similarly computed at the 
TABLE 2: Generator data and demand data.

\begin{tabular}{|c|c|c|c|c|c|c|c|c|}
\hline Bus & $p_{G_{i}}^{\max }$ & $p_{G_{i}}^{\min }$ & $q_{G_{i}}^{\max }$ & $q_{G_{i}}^{\min }$ & $a_{i}$ & $b_{i}$ & $p_{D_{i}}$ & $q_{D_{i}}$ \\
\hline 1 & 132.5 & 112.5 & 150.0 & -150.0 & 8.5 & 0.0005 & 0.0 & 0.0 \\
\hline 2 & 165.0 & 140.0 & 150.0 & -150.0 & 9.0 & 0.0005 & 0.0 & 0.0 \\
\hline 3 & 80.0 & 60.0 & 150.0 & -150.0 & 9.5 & 0.0005 & 0.0 & 0.0 \\
\hline 4 & - & - & - & - & - & - & 120.0 & 80 \\
\hline 5 & - & - & - & - & - & - & 115.0 & 82.0 \\
\hline 6 & - & - & - & - & - & - & 104.0 & 66.0 \\
\hline
\end{tabular}

TABLE 3: Line data.

\begin{tabular}{lcccc}
\hline Line & $R_{i j}$ & $X_{i j}$ & $B_{i j}^{S}$ & $S_{i j}^{\max }$ \\
\hline $1-2$ & 0.10 & 0.20 & 0.04 & 36.0 \\
$1-4$ & 0.05 & 0.20 & 0.04 & 72.0 \\
$1-5$ & 0.08 & 0.30 & 0.06 & 63.6 \\
$2-3$ & 0.05 & 0.25 & 0.06 & 36.0 \\
$2-4$ & 0.05 & 0.10 & 0.02 & 91.2 \\
$2-5$ & 0.10 & 0.30 & 0.04 & 42.0 \\
$2-6$ & 0.07 & 0.20 & 0.05 & 72.0 \\
$3-5$ & 0.12 & 0.26 & 0.05 & 36.0 \\
$3-6$ & 0.02 & 0.10 & 0.02 & 84.0 \\
$4-5$ & 0.20 & 0.40 & 0.08 & 18.0 \\
$4-6$ & 0.10 & 0.30 & 0.06 & 14.4 \\
\hline
\end{tabular}

same time. We present the local sensitivity formulas of LMPs for both the cases of regular nondegenerate and the cases of regular degenerate solutions. So the main conclusion is that it can consider the sensitivity analysis in the broader sense; a simple matrix form of the sensitivities for the LMPs with respect to all parameters is given. Numerical results are also given to show that this method is effective.

\section{Conflict of Interests}

The authors declare that there is no conflict of interests regarding the publication of this paper.

\section{Acknowledgments}

This work is supported by the construct program of the key discipline in Hunan Province, National Natural Science Foundation of China (11301445), the Research Foundation of Education Bureau of Hunan Province (13B121), and the Social Science Fund Project of Hunan Province (11YBA300).

\section{References}

[1] 2013, http://www.iso-ne.com.

[2] http://www.nyiso.com, 2013.

[3] 2013, http://www.pjm.com/.

[4] X. Kai, S. Yonghua, Y. Erkeng, and L. Guangyi, "Optimal power flow based spot pricing algorithm via interior point methods: on the economic meanings of $\lambda \mathrm{p}$ and $\lambda \mathrm{q}$," Automation of Electric Power Systems, vol. 23, no. 2, pp. 5-10, 1999.
[5] A. M. de Souza, V. A. de Sousa, and G. R. M. da Costa, "Studies of cases in power systems by sensitivity analysis oriented by OPF,' International Journal of Electrical Power and Energy Systems, vol. 32, no. 9, pp. 969-974, 2010.

[6] A. J. Conejo, E. Castillo, R. Mínguez, and F. Milano, "Locational marginal price sensitivities," IEEE Transactions on Power Systems, vol. 20, no. 4, pp. 2026-2033, 2005.

[7] C. Murillo-Sanchez, S. Ede, T. Mount, R. Thomas, and R. Zimmerman, "An engineering approach to monitoring market power in restructured for electricity," in Proceedings of the 24th IAEE Annual International Conference, International Association Energy Economics, Houston, Tex, USA, April 2001.

[8] P. R. Gribik, D. Shirmohammadi, S. Hao, and C. L. Thomas, "Optimal power flow sensitivity analysis," IEEE Transactions on Power Systems, vol. 5, no. 3, pp. 969-976, 1990.

[9] R. Baldick, "Variation of distribution factors with loading," IEEE Transactions on Power Systems, vol. 18, no. 4, pp. 1316-1323, 2003.

[10] A. Fradi, S. Brignone, and B. F. Wollenberg, "Calculation of energy transaction allocation factors," IEEE Transactions on Power Systems, vol. 16, no. 2, pp. 266-272, 2001.

[11] E. Castillo, A. J. Conejo, C. Castillo, R. Minguez, and D. Ortigosa, "Perturbation approach to sensitivity analysis in mathematical programming," Journal of Optimization Theory and Applications, vol. 128, no. 1, pp. 49-74, 2006.

[12] A. V. Fiacco, Introduction to Sensitivity and Stability Analysis in Nonlinear Programming, Academic Press, Orlando, Fla, USA, 1983.

[13] D. Shirmohammadi and C. L. Thomas, "Valuation of the transmission impact in a resource bidding process," IEEE Transactions on Power Systems, vol. 6, no. 1, pp. 316-323, 1991.

[14] L. Wang and M. Mazumdar, "Using a system model to decompose the effects of influential factors on locational marginal prices," IEEE Transactions on Power Systems, vol. 22, no. 4, pp. 1456-1465, 2007.

[15] Q. Zhou, L. Tesfatsion, and C. Liu, "Global sensitivity analysis for the short-term prediction of system variables," in Proceedings of the Power and Energy Society General Meeting (PES '10), August 2010.

[16] L. Yang, Y. Chen, and X. Tong, "A note on local sensitivity analysis for parametric optimization problem," Pacific Journal of Optimization, vol. 8, no. 1, pp. 185-195, 2012.

[17] A. Fischer, "A Newton-type method for positive-semidefinite linear complementarity problems," Journal of Optimization Theory and Applications, vol. 86, no. 3, pp. 585-608, 1995.

[18] L. Q. Qi and J. Sun, "A nonsmooth version of Newton's method," Mathematical Programming, vol. 58, no. 3, pp. 353-367, 1993. 


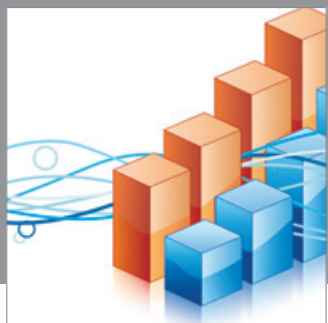

Advances in

Operations Research

mansans

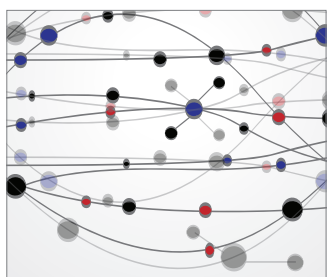

The Scientific World Journal
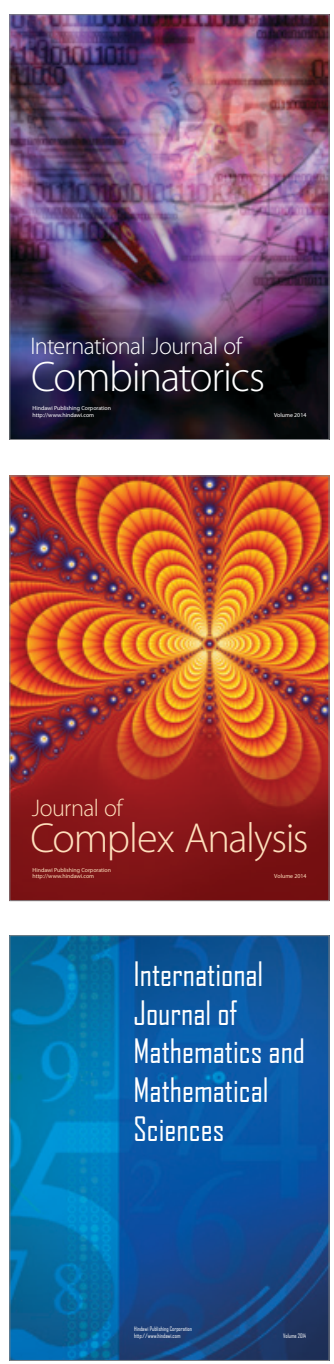
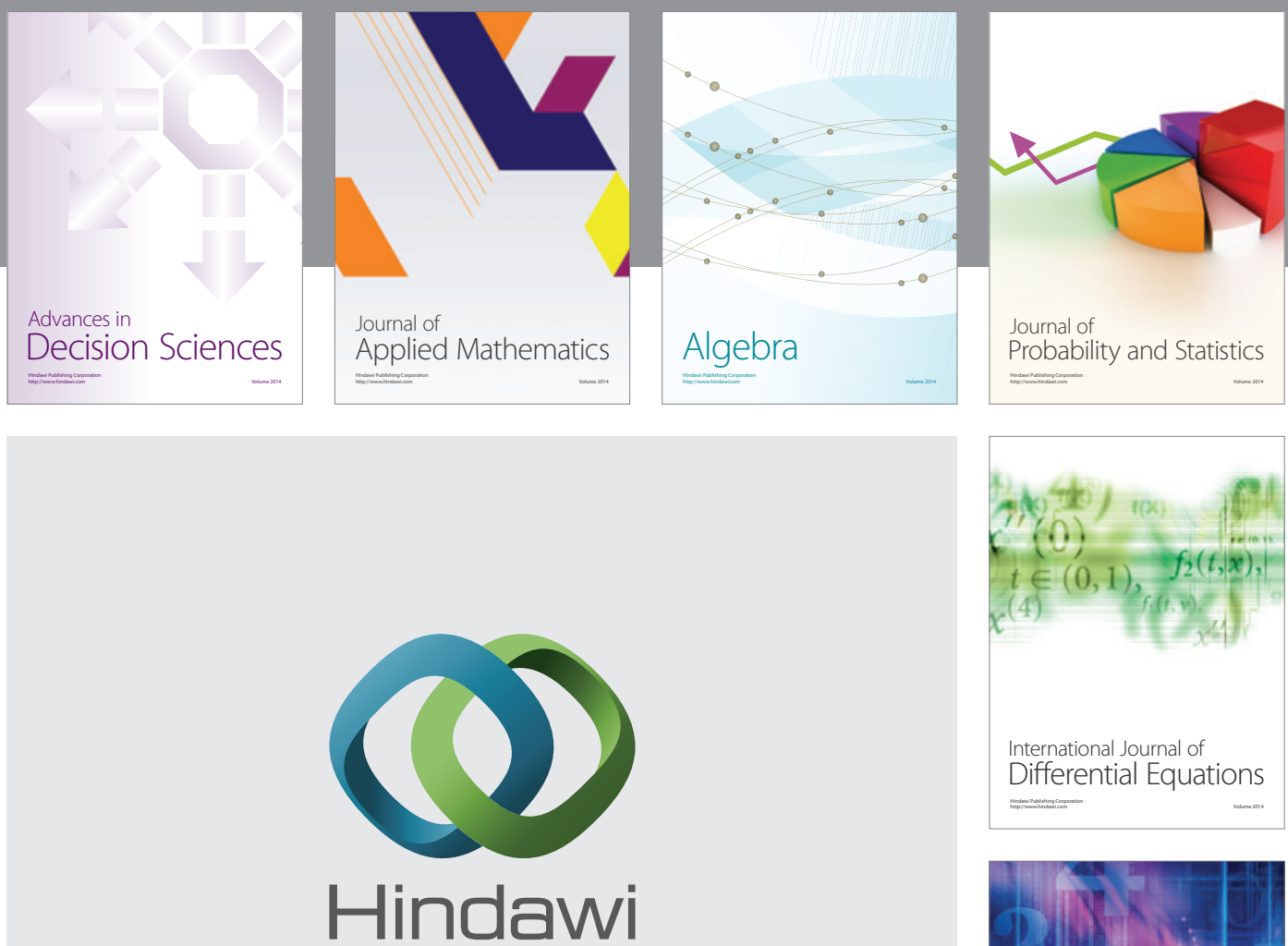

Submit your manuscripts at http://www.hindawi.com
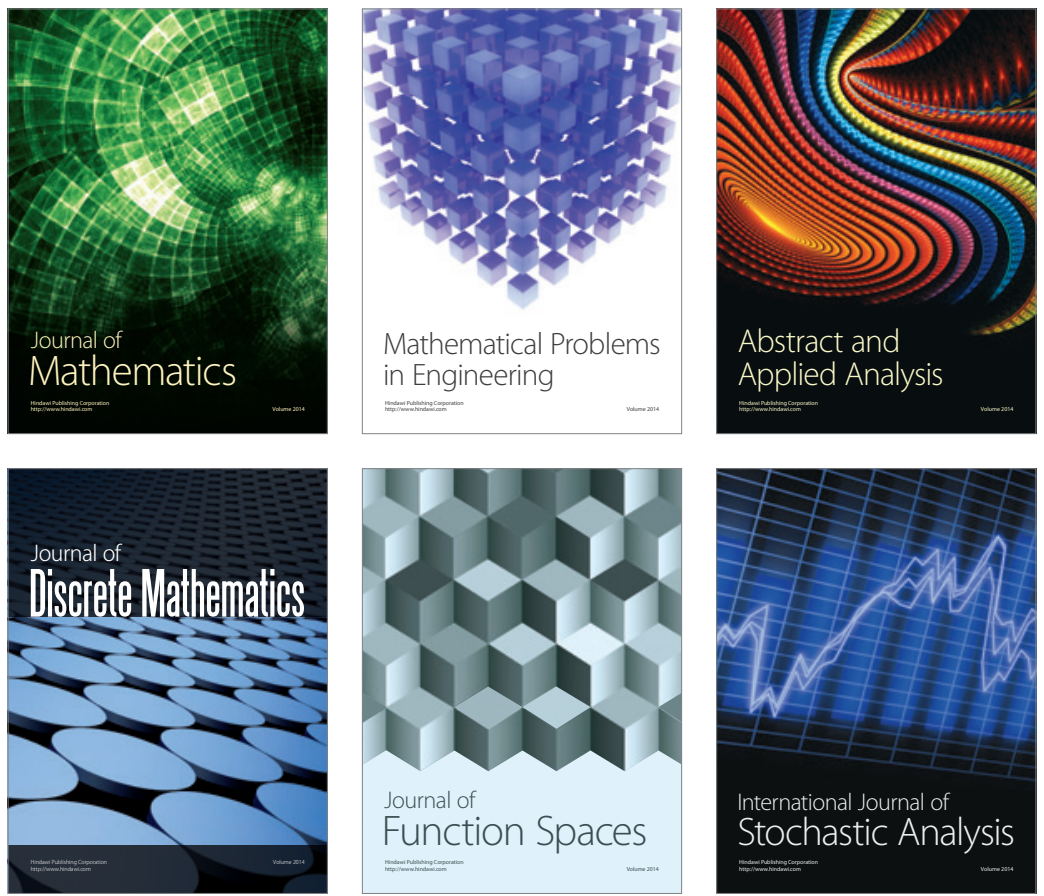

Journal of

Function Spaces

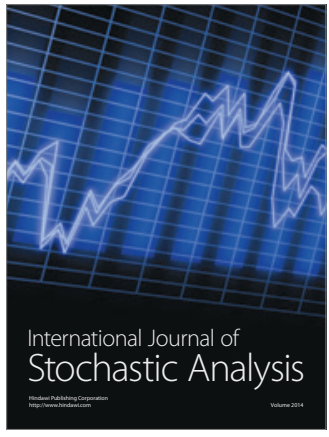

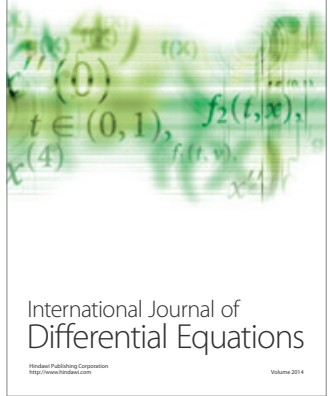
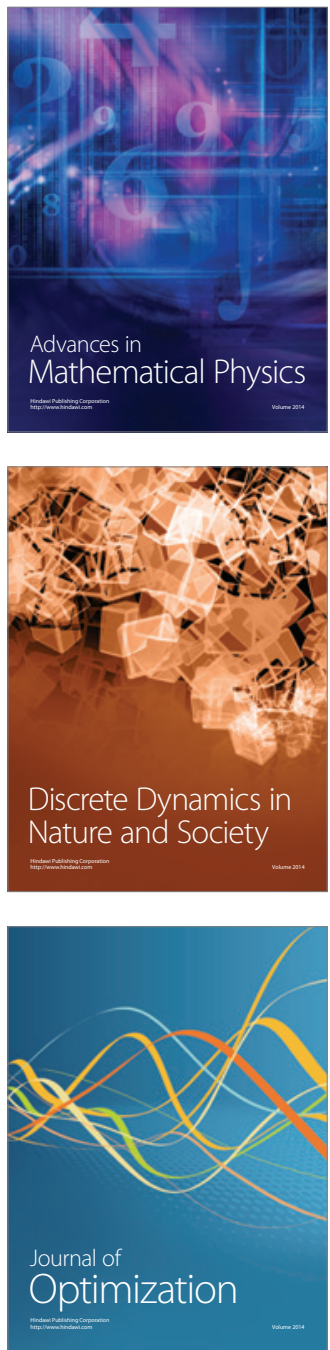